\title{
The Effects of Adjuvant Chemoradiotherapy on Survival in Patients with Primary Laryngeal Cancer with Close Surgical Margins and Positive Surgical Margins
}

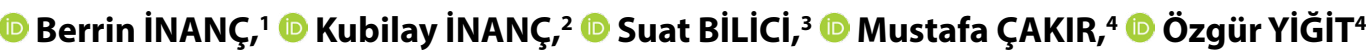 \\ 'Department of Radiation Oncology, İstanbul Research and Education Hospital, İstanbul-Turkey \\ 2Department of Radiation Oncology, Şişli Hamidiye Etfal Research and Education Hospital, İstanbul-Turkey \\ ${ }^{3}$ Department of Otorhinolaryngology, Acıbadem University Faculty of Medicine, İstanbul-Turkey \\ ${ }^{4}$ Department of Otorhinolaryngology, İstanbul Research and Education Hospital, İstanbul-Turkey
}

\begin{abstract}
OBJECTIVE
The gold standard treatment for squamous cell carcinoma of the larynx is a resection of the primary tumour with negative surgical margins. In this study, we retrospectively investigated the effects of adjuvant concurrent chemoradiation on the survival rates of laryngeal cancer with close surgery margins and patients with positive surgery margin.
\end{abstract}

\section{METHODS}

A total of 40 patients treated with concurrent chemoradiation were included in this study. All of these patients had surgery for laryngeal cancer and had received $66 \mathrm{~Gy}$ (2Gy/fr) of radiotherapy and concurrent weekly cisplatin. Patients were stratified into two groups according to surgical margin status. Members of group 1 had a positive surgery margin; group 2 patients had close surgical margins and were studied for comparative analyses.

\section{RESULTS}

At the median follow-up of 40 months, nine patients (44.4\%) experienced local/regional failures, six of which were detected on the second follow-up. In patients with positive surgical margin, overall survival (OS), disease-free survival (DFS), and loco-regional progression-free survival (LRPFS) were 59\%, 44\% and $81 \%$, respectively. OS, DFS and LRPFS for those with close surgical margins were $57 \%, 46 \%$ and $71 \%$, respectively. No statistically significant differences related to OS, DFS, and LRPFS were observed between groups 1 and 2 ( $\mathrm{p}=0.802, \mathrm{p}=0.610$ and $\mathrm{p}=0.383$, respectively). On univariate Cox-regression analysis, the presence of perineural invasion and lymphovascular invasion was statistically significant for OS and DFS ( $p=<0.05)$. Being 65 years old or above was statistically significant for OS $(p=<0.05)$.

\section{CONCLUSION}

Although limited by small sample size, our results revealed that there was no significant difference between close and positive margins in terms of OS, DFS and LRFS. More detailed and comprehensive studies on the close surgical margin $(2 \mathrm{~mm}, 3 \mathrm{~mm}$ and $4 \mathrm{~mm}$ ) are needed.

Keywords: Concurrent chemoradiotherapy; close surgical margin; laryngeal cancer; positive surgical margin. Copyright $\odot$ 2020, Turkish Society for Radiation Oncology

Dr. Berrin INANÇ

Istanbul Araştırma ve Eğitim Hastanesi,

Radyasyon Onkolojisi Kliniği,

Istanbul-Turkey

E-mail: byalcin77@hotmail.com 


\section{Introduction}

Laryngeal cancer is the most common cancer of the respiratory tract. Surgery, radiotherapy (RT) and chemotherapy (CT) are the most important modalities in the treatment of laryngeal cancers. As indicated by the pathology of the disease, the goal of surgery for laryngeal cancer is the complete resection of the tumour with negative margins. Positive margins and close surgical margins increase the risk of local relapse, cause a decrease in survival rates, and require postoperative adjuvant treatment.[1,2] Both clinical and pathological studies show the importance of the relationship between local tumour recurrence and close and positive margins.[3] Thus, the first proposal for patients with positive surgical margins or close margins the expansion of the surgery so that no tumour remains at or near the surgical margins. However, extended surgery is not possible for every patient. Extended surgery may bring the risk of additional morbidity and anatomical difficulties in the head and neck regions. Instead, sequential or concurrent chemoradiotherapy (C-CRT) is applied to patients who do not undergo re-excision for close surgical margins or positive margins. [4,5]

In this study, we examined whether a significant difference exists between patients with close surgical margins and patients' positive surgical margins who received adjuvant C-CRT We looked specifically at LRPRS, DFS and overall survival.

\section{Materials and Methods}

Between January of 2011 and December of 2016, 40 patients who had been treated at were included in Istanbul Education and Research Hospital this study. This study was approved by the local ethics committee of the University of Health Science, Istanbul Education and Research Hospital, Turkey, Human Research Ethics Committee (approval number: H-2018-1431) according to Helsinki declaration, and informed consent was obtained from all patients after a thorough explanation of the study. These patients had resectable laryngeal cancer with histological proof of malignancy and were retrospectively enrolled. Surgical specimen was examined for each enrolled. A positive margin was considered to mean that the specimen showed the presence of the tumor, and the tumor was within $1 \mathrm{~mm}$ of the surgical border. The specimens that had a surgical margin of $2 \mathrm{~mm}$ to $3 \mathrm{~mm}$ were considered to have close margins. All of these patients received C-CRT. We referenced several prognostic variables, includ- ing age (continuous), gender (male or female), cancer stage -according to the American Joint Committee on Cancer $7^{\text {th }}$ edition [6]-,operation type, tumor diameter, histological grade, lymphovascular invasion (LVI), perineural invasion (PNI) with survival. Because the study population was small, and because the exact prognostic values of these parameters were not clearly specified, we chose to set the selection method based on surgical margins. Patients were categorized into two groups: Group 1 (positive surgical margins group) and Group 2 (close surgical margins group) and were then compared by LRPFS, DFS, and OS. LRPFS was defined as survival without local/regional failure, calculated as the time between the first day of treatment and the date of local/regional failure or death/last visit. DFS and OS were calculated as the time between the first day of treatment and any type of disease progression, and the date of death/last visit, respectively.

\section{Treatment Planning and Treatment Delivery \\ Surgery}

The surgical suitability of all patients was determined in the multidisciplinary head and neck team. For determining the type of surgeon, the team considered the primary site, the prevalence of the disease and the cosmetic results, and complete tumour resection was the established goal.

\section{RT Planning}

Patients were immobilized in a supine position with a thermoplastic mask. An enhanced computed tomography scan was acquired with IV plus contrast media through $3 \mathrm{~mm}$ slices from the skull base to the diaphragm inferior border. A standardized protocol with $140 \mathrm{kV} / 80 \mathrm{~mA}$ was used. One radiation oncologist defined the target volumes and contoured the clinical target volume (CTV), planning target volume (PTV) and organs at risk (OARs).

PTV was defined as CTV $+5 \mathrm{~mm}$ in each direction, allowing for microscopic extension and setup errors. The treatment was planned in accordance with the ICRU-83 guidelines.[7]

The three target volume was irradiated via the simultaneous integrated boost technique with dynamic intensity-modulated radiation therapy (IMRT). Patients received RT regimens using $6 \mathrm{MV}$ photon energy linear accelerators. A dose of $66 \mathrm{~Gy}(200 \mathrm{~Gy} / \mathrm{fr}, 5$ days/ week) was administered to the operative area, which was a region having either a positive or close surgical margin. A dose of $60 \mathrm{~Gy}$ ( $200 \mathrm{~Gy} / \mathrm{fr}, 5$ days/week) was administered to the involved lymph nodes region. A 
dose of 54 Gy (180 Gy/fr, 5 days/week) was administered to the uninvolved lymph nodes region. Isodose curves were not cooler than $95 \%$ or hotter than $107 \%$. Dose-volume histograms assessed each patient's target volume coverage and organ-at-risk doses. For normal tissues, maximum dose limits were $45 \mathrm{~Gy}$ for the spinal cord, 45 Gy for the oral cavity, 54 Gy for the brainstem, and 26 Gy for the parotid glands. Dose levels beyond these limits were considered as exclusion criteria.

\section{Chemotherapy}

Patients received weekly cisplatin $\left(40 \mathrm{mg} / \mathrm{m}^{2} /\right.$ week $)$ throughout the RT course as a radiosensitizer.

\section{Response Evaluation and Follow-up}

During C-CRT, patients were evaluated at least once a week with a clinical examination, and all patients' blood counts and biochemistries were required. The treatment responses were assessed by endoscopic examination for the first control and were performed two weeks after the treatment. Subsequent controls included physical examinations and endoscopic and radiological imaging every three months. Follow-ups were arranged every three months for the first two years and every six months for years three through five. During the follow-up period, a magnetic resonance imaging (MRI) or 18F-fluoro-deoxy-D-glucose positron emission tomography (FDG-PET) examination was requested in patients with suspected local/ regional recurrence and metastasis. Loco-regional recurrences and metastasis suspicions were verified with biopsies. The decision to request biopsy and additional imaging modality (FDG-PET) for these patients was determined by local MDT (multidisciplinary team). Then, a surgeon was selected for re-excision. Patients who underwent re-excision and metastasized were referred to the Department of Medical Oncology for systemic therapy.

\section{Statistical Analysis}

The descriptive statistics included the mean, standard deviation, median, minimum, maximum, frequency, and ratio values of the data. Survival analysis was performed using the Kaplan-Meier method. A multivariate-univariate analysis (Cox regression analysis) was used to evaluate the independent risk factors that affected survival. Effect size or hazard ratio (HR) and 95\% CI were used to report the magnitude of difference. All analyses were performed using statistical software IBM SPSS ver. 22.0 (IBM Co., Chicago, USA). P< 0.05 was considered significant.

\section{Results}

Sixty five patients were enrolled, and 40 were eligible. Twenty-six patients were positive for surgical margin, and fourteen patients were close surgical margin. Concerning Cancer Staging, T2:21 patients, T3:9 patients, T4:10 patients and N0:21 patients, N1:9 patient and N2b:10. Patient characteristics were as depicted in Table 1. Almost all the patients well tolerated C-CRT, because cisplatin given weekly. No CT dose reduction was necessary, but treatment was interrupted in 3 cases (13.3\%). These patients required hospital admission for the management of their acute toxicities. The incidence of grade 3 or 4 toxicities: mucositis (33\%), dermatitis (41\%), dysphagia (40\%), mouth/neck pain (20\%) and neutropenia (2\%).

At a median follow-up of 40 months (range: 1672 ). 14 of 40 patients (35\%) were dead. Nine patients (22.5\%) developed field recurrences, six of which were positive margins group, and three of which were close margins group. While most of the recurrence in the operation field, only two of them were observed in neck lymph nodal field. Distant relapses without local failures were encountered in 11 (27.5\%) patients and all of them were positive surgical margins. Distant failure site included the lung.

The OS rate were $57 \%(n=14)$ at patient with group: 1 and $59 \%(n=26)$ at patients with group: 2 . There was no statistically significant difference between concerning OS rate ( $\mathrm{p}=0.802$, Fig. 1$)$. In the univariate cox-regression analysis, gender, stage, operation type, histologic grade, tumour diameter, patients with close and positive surgical margins did not observe a significant effect on overall survival time ( $>0.05$, Table 2 ). In a multivariate Cox-regression analysis, the presence of perineural invasion (PNI), lymphovascular invasion

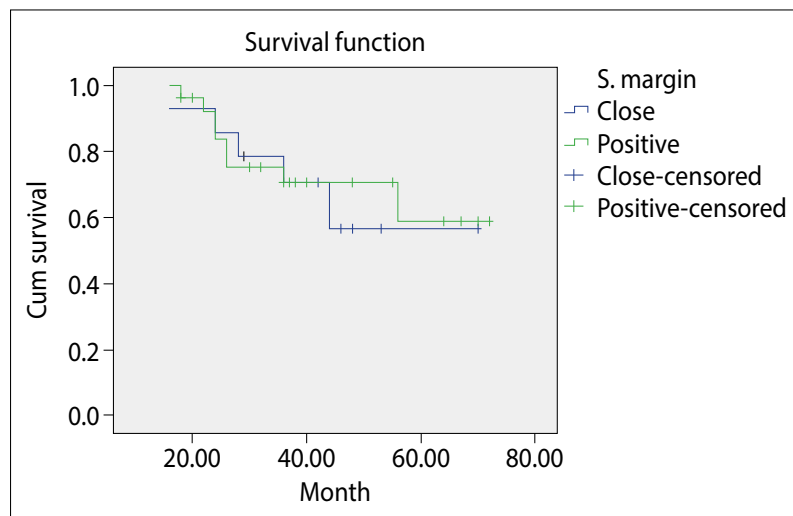

Fig. 1. Overall survival curve. 
Table 1 Patient characteristics

\begin{tabular}{|c|c|c|c|c|}
\hline & Min-Max & Med & \multicolumn{2}{|c|}{ Mean \pm SD $/ n-\%$} \\
\hline Age & $49-76$ & 62 & \multicolumn{2}{|c|}{$60.6 \pm 7.4$} \\
\hline \multicolumn{5}{|l|}{ Gender } \\
\hline Female & & & 3 & $7.5 \%$ \\
\hline Male & & & 37 & $92.5 \%$ \\
\hline Tumor size & $1-6$ & 3 & & \\
\hline \multicolumn{5}{|l|}{ Tumor stage } \\
\hline II & & & 12 & $30 \%$ \\
\hline III & & & 15 & $37.5 \%$ \\
\hline IV & & & 13 & $32.5 \%$ \\
\hline \multicolumn{5}{|l|}{ Operation Type } \\
\hline Total Laryngectomy+N.D & & & 18 & $45.0 \%$ \\
\hline Partial Laryngectomy+N.D & & & 7 & $17.5 \%$ \\
\hline Supraglotic Laryngectomy+N.D & & & 9 & $22.5 \%$ \\
\hline Supracriciod Laryngectomy+N.D & & & 6 & $15.0 \%$ \\
\hline \multicolumn{5}{|l|}{ Histological Grade } \\
\hline 1 & & & 2 & $5.0 \%$ \\
\hline II & & & 29 & $72.5 \%$ \\
\hline III & & & 9 & $22.5 \%$ \\
\hline \multicolumn{5}{|l|}{ Lymphovascular Invasion } \\
\hline$(-)$ & & & 30 & $75.0 \%$ \\
\hline$(+)$ & & & 10 & $25.0 \%$ \\
\hline \multicolumn{5}{|l|}{ Perineural Invasion } \\
\hline$(-)$ & & & 30 & $75.0 \%$ \\
\hline$(+)$ & & & 10 & $25.0 \%$ \\
\hline \multicolumn{5}{|l|}{ Surgical Margin } \\
\hline$(-)$ & & & 14 & $35.0 \%$ \\
\hline$(+)$ & & & 26 & $65.0 \%$ \\
\hline Follow Time & $16-72$ & 37 & \multicolumn{2}{|c|}{$40.0 \pm 16.0$} \\
\hline
\end{tabular}

ND: Neck dissection

(LVI) and being 65 years old and above was found to be significant concerning overall survival $(\mathrm{p}<0.009$, Hazard ratio (HR): 4.63, 95\% Confidence interval (CI): 1.46-14.86 and $\mathrm{p}=0.051$, HR: 3.01, CI: 0.99-9.14, respectively).

The DFS rate was $44 \%$ in patients with close surgical margins and $46 \%$ in patients with positive surgical margins. There was no statistically significant difference between concerning DFS rate ( $\mathrm{p}=0.610$, Fig. 2). Median DFS was 17 month in the close margin group, and 27 months in positive margin group. There was no significant effect on DFS concerning the age, gender, stage, operation type, histologic grade, tumour diameter and close surgical margins and positive surgical margins in the univariate model $(\mathrm{p}>0.05)$. The presence of LVI and PNI were statistically significant in both patients with close and positive surgical margins $(\mathrm{p}<0.05)$. Both parameters had significant differences in univariate regression analysis $(p=0.047$ and

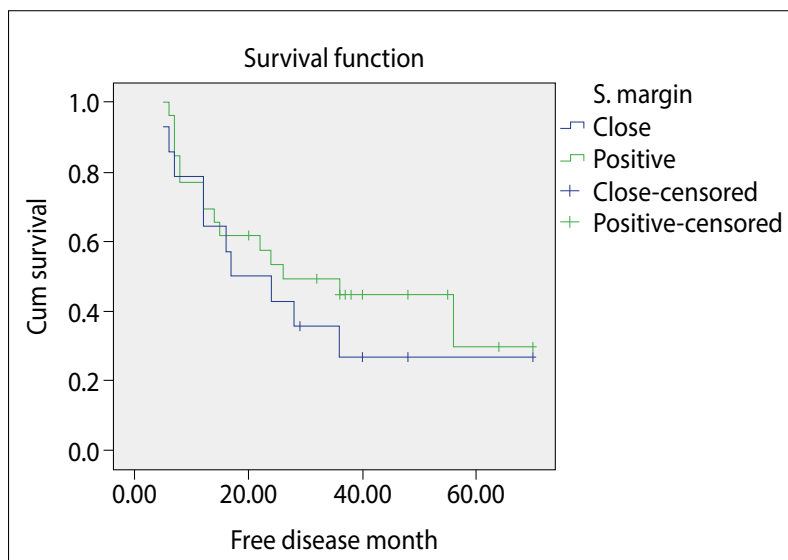

Fig. 2. Disease-free survival curve.

$\mathrm{p}=0.004$ ) (Table 2). In a multivariate Cox-regression analysis, PNI was statistically significant $(\mathrm{p}=0.007, \mathrm{HR}$ : 3.25, 95\% CI: 1.37-7.70). 
Table 2 Univariate Kaplan-Meier analysis for OS and DFS

\begin{tabular}{|c|c|c|c|c|c|c|c|c|}
\hline \multirow[t]{2}{*}{$\begin{array}{l}\text { Univariate analysis } \\
\text { (Kaplan-Meier) }\end{array}$} & \multicolumn{5}{|c|}{$\begin{array}{l}\text { Overall survival } \\
\text { (OS) }\end{array}$} & \multicolumn{3}{|c|}{$\begin{array}{l}\text { Disease-free survival } \\
\text { (DFS) }\end{array}$} \\
\hline & $\begin{array}{l}\text { Patient } \\
\text { number }\end{array}$ & 5 years & 3 years (\%) & $\begin{array}{l}\text { Median survival } \\
\text { (month) }\end{array}$ & $\mathbf{p}$ & 3 years $(\%)$ & $\begin{array}{c}\text { Median survival } \\
\text { (month) }\end{array}$ & $\mathbf{p}$ \\
\hline \multicolumn{9}{|l|}{ Surgical margin } \\
\hline Close & 14 & 57 & 71 & - & 0.802 & 27 & 17 & 0.429 \\
\hline Positive & 26 & 59 & 71 & - & & 45 & 26 & \\
\hline \multicolumn{9}{|l|}{ Stage } \\
\hline II & 12 & 44 & 67 & 56 & 0.599 & 42 & 26 & 0.836 \\
\hline III & 29 & 78 & 93 & - & & 60 & 36 & \\
\hline IVA & 13 & 56 & 67 & - & & 31 & 17 & \\
\hline \multicolumn{9}{|l|}{ Grade } \\
\hline I & 2 & - & - & - & 0.586 & 50 & 17 & 0.660 \\
\hline II & 29 & 47 & 78 & - & & 48 & 48 & \\
\hline III & 9 & 67 & 67 & & & 33 & 12 & \\
\hline \multicolumn{9}{|l|}{ LVI } \\
\hline No & 30 & 67 & 78 & - & 0.047 & 49 & 36 & 0.047 \\
\hline Yes & 10 & - & 50 & 36 & & 10 & 15 & \\
\hline \multicolumn{9}{|l|}{$\mathrm{PNI}$} \\
\hline No & 30 & 68 & 86 & - & 0.002 & 56 & 36 & 0.004 \\
\hline Yes & 10 & - & 45 & 28 & & - & 12 & \\
\hline \multicolumn{9}{|l|}{ Age } \\
\hline 65 years below & 29 & 77 & 82 & - & 0.022 & 43 & 28 & 0.273 \\
\hline 65 years above & 11 & - & 55 & 44 & & 27 & 17 & \\
\hline
\end{tabular}

LVI: Lymphovascular invasion; PNI: Perineural invasion

LRFS ratio was $71 \%$ in the patients' close margin group, and $81 \%$ in the patient's positive margin group. There was no statistical difference between the two groups ( $\mathrm{p}=0.383)$. Cox-regression could not be performed because a significant variable could not be obtained in univariate analysis.

\section{Discussion}

The most important goal of the oncologic surgeon is pathologically a complete resection with a negative surgical margin. The first proposal in patients with close surgical margins or positive surgical margins is the expansion of the surgery so that no tumor remains in or close surgical margin; however, this new surgical procedure is not possible for every patient, mainly due to additional morbidity and anatomical difficulties in the head and neck region. C-CRT is applied to these patients for close surgical margins or positive. The aim of this rational additional treatment is increasing radio-sensitivity, providing local control and eradication of systemic micro-metastasis. [8] The positivity of surgical margin and ECE (extra- capsular invasion) are most important indications for postoperative adjuvant treatment.[5] In this study, we not consider the presence of ECE, because it will not change our adjuvant treatment decision. The current limit for positive surgical margin is $0.5 \mathrm{~cm}$.[9] In some studies, the surgical margin was examined in three different groups. $[3,10]$

1. Free surgical margin: absence of tumour around 5 mm border

2. Close to surgical margin: tumour present in the region containing $5 \mathrm{~mm}$ of borderline (1-5 $\mathrm{mm})$; but there is no tumour at border.

3. Positive surgical margin: border tumour present or $<1 \mathrm{~mm}$

Close margin or the appropriate margin clearance from tumor remains as a controversial debate in the literature.

A microscopically positive and close margin is associated with a higher risk for local recurrence and poor clinical outcome. Sutton et al. reported that local recurrence rates found as $55 \%$ in oral squamous cell carcinomas with positive surgical margin and 33\% in the close surgical margin.[3] In the present study, 
patients with positive and close surgical margins had significantly lower local recurrences rates (19\%, 29\%), respectively. Sutton et al. performed alone adjuvant RT in their study. However, we performed adjuvant C-CRT in all patients. This data may suggest that chemotherapy will play a decisive role in survival in patients with positive and close surgical margins. In our study, we did not difference between stage II and IV because most of the stage II patients were surgical margin positive patients ( 9 of 12 patients). The status of surgical margins is one of the most important prognostic factors for survival in patients with head and neck cancer. In the literature, various studies supported these findings.[11-13]

Two prospective, randomized clinical trials that used in the decision making about adjuvant RT and CRT for squamous cell cancer of the head and neck included margin status as their selection criteria. The Radiation Therapy Oncology Group (RTOG) 9501 study only included patients with positive margins, whereas the European Organization for Research and Treatment of Cancer Trial (EORTC) 22931 study also included patients with a close margin, defined as less than $5.0 \mathrm{~mm} .[14]$

In EORTC-22931, OS was 53\% in C-CRT group and DFS was $47 \%$ in C-CRT group. In our study, OS was $59 \%$ in the positive margin group and $57 \%$ in the close margin group. In addition, DFS was $44 \%$ in the positive margin group and $46 \%$ in a close margin group. Our results were found to be similar to literature. There was no significant difference.

We compared the local control rates found higher in both groups (group 1: $81 \%$ vs. group 2:71\%). There was no difference between $2-3 \mathrm{~mm}$ and positive margin at the LRPFS. Zanoni et al. reported that a surgical margin of $2.2 \mathrm{~mm}$ was optimal to predict for loco-regional recurrence-free survival.[15] Patients with a margin between 2.3 and $5 \mathrm{~mm}$ or above $5 \mathrm{~mm}$ had similar outcomes. Tumors originating at different sites behave very differently. This study included patients with squamous cell carcinoma of the oral cavity. Our series are small size and all our patients with larynx cancer. Additional randomized studies are necessary to confirm these results further.

\section{Limitations}

In all the above mentioned clinical study, the reason why few percent (around 10-15\%) of patients presented local relapse is unknown and could have nothing to do with an inadequate resection margin, but rather with unfavorable tumour biology.

\section{Conclusion}

In this study, because both groups received the same treatment, there was no significant difference between the two groups in terms of OS, DFS, and LRFS. Other clinical and pathological prognostic factors should also be taken into consideration when recommending further treatment.

\section{Abbreviations}

RT: Radiotherapy

C-CRT: Concurrent chemoradiotherapy

IMRT: Intensity- modulated radiotherapy

FDG-PET: 18F-fluoro-deoxy-D-glucose positron emission tomography

MRI: Magnetic resonance imaging

CT: Computerized tomography

PTV: The planning target volume

CTV: The clinical target volume

OARs: Organ at risk

MV: Million volts

LVI: Lymphovascular invasion

PNI: Perineural invasion

MDT: Multidisciplinary Team

Peer-review: Externally peer-reviewed.

Conflict of Interest: The authors declare that they have no conflict of interest.

Ethics Committee Approval: This study was approved by the local ethics committee of the University of Health Science, Istanbul Education and Research Hospital, Turkey, Human Research Ethics Committee (approval number: H-2018-1431).

Financial Support: Not applicable.

Authorship contributions: Concept - B.I.j; Design - B.I.;; Supervision - B.İ.; Funding - None; Materials - B.İ., S.B., M.Ç.; Data collection and/or processing - B.İ.; Data analysis and/or interpretation - S.B., M.Ç., Ö.Y.; Literature search K.I.; Writing - B.I..; Critical review - Ö.Y.

\section{References}

1. Shang J, Gu J, Han Q, Xu Y, Yu X, Wang K. Chemoradiotherapy is superior to radiotherapy alone after surgery in advanced squamous cell carcinoma of the head and neck: a systematic review and meta-analysis. Int J Clin Exp Med 2014;7(9):2478-87.

2. Bernier J1, Domenge C, Ozsahin M, Matuszewska K, Lefèbvre JL, Greiner RH, et al. Postoperative Irradia- 
tion with or without concomitant chemotherapy for locally dvanced head and neck cancer. N Engl J Med. 2004;350(19):1945-52.

3. Sutton DN, Brown JS, Rogers SN, Vaughan ED, Woolgar JA. The prognostic implications of the surgical margin in oral squamous cell carcinoma. Int J Oral Maxillofac Surg 2003;32(1):30-4.

4. Al-Sarraf M. Treatment of locally advanced head and neck cancer: Historical and critical review. Cancer Control 2002;9(5):387-99.

5. Shah JP, Cendon RA, Farr HW, Strong EW. Carcinoma of the oral cavity. Factors affecting treatment failure at the primary site and neck. Am J Surg 1976;132(4):504-7.

6. Edge S, Byrd DR, Conpton CC, Fritz AG, Grene FL, Trotti A, editors. AJCC cancer staging manual. $7^{\text {th }} \mathrm{ed}$. New York, USA: Springer Verlag; 2010.

7. Prescribing, recording and reporting photon-beam intensity-modulated radiation therapy IMRT. International Commission on Radiation Units and Measurements (ICRU) 2010;10(1):83.

8. Brizel DM, Esclamado R. Concurrent chemoradiotherapy for locally advanced, nonmetastatic squamous carcinoma of the head and neck: consensus, controversy, and conundrum. J Clin Oncol 2006;24(17):2612-7.

9. Looser KG, Shah JP, Strong EW. The significance of positive margins in surgically resected epidermoid carcinomas. Head Neck Surg 1978;1(2):107-11.
10. Batsakis JG. Surgical excision margins: a pathologist's perspective. Adv Anat Pathol. 1999;6(3):140-8.

11. Smid L, Budihna M, Zakotnik B, Soba E, Strojan P, Fajdiga I, et al. Postoperative concomitant irradiation and chemotherapy with mitomycin $\mathrm{C}$ and bleomycin for advanced head-and-neck carcinoma. Int J Radiat Oncol Biol Phys 2003;56(4):1055-62.

12. Cooper JS, Pajak TF, Forastiere AA, Jacobs J, Campbell BH, Saxman SB, et al. Radiation Therapy Oncology Group 9501/ Intergroup. Postoperative concurrent radiotherapy and chemotherapy for high-risk squamous cell carcinoma of the head and neck. N Engl J Med 2004;350(19):1937-44.

13. Brizel DM, Esclamado R. Concurrent chemoradiotherapy for locally advanced, nonmetastatic squamous carcinoma of the head and neck: consensus, controversy, and conundrum. J Clin Oncol 2006;24(17):2612-7.

14. Bernier J, Cooper JS, Pajak TF, van Glabbeke M, Bourhis J, Forastiere A, et al. Defining risk levels in locally advanced head and neck cancers:a comparative analysis of concurrent postoperative radiation plus chemotheraphy trials of the EORTC(22931) and RTOG(9501). Head Neck 2005;27(10):843-50.

15.Zanoni DK, Migliacci JC, Xu B, Katabi N, Montero $\mathrm{PH}$, Ganly I, et al. A proposal to revisit the definition of close margin is squamous cell carcinoma of oral tongue. JAMA Otolaryngol Head Neck Surg 2017;143(6):555-60. 